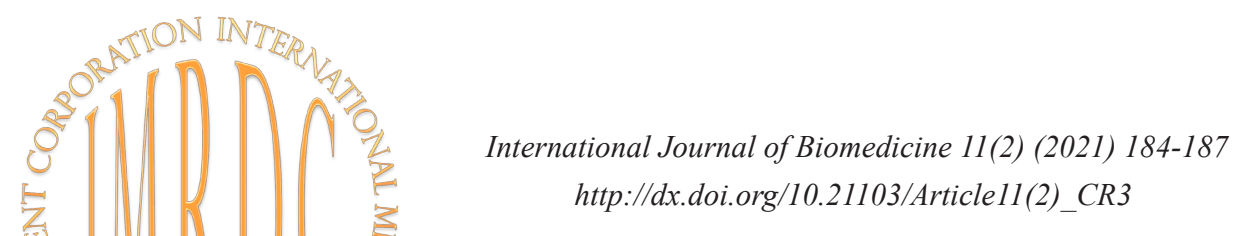

INTERNATIONAL JOURNAL OF BIOMEDICINE

\title{
Endodontic Treatment of Periapical Lesions Using Bioceramic Sealers
}

\author{
Bogdan R. Shumilovich, PhD, ScD; Anna V. Podoprigora, PhD, ScD*; \\ Andrey V. Sushchenko, PhD, ScD; Vladimir V. Rostovtsev, PhD; \\ Irina S. Bishtova, PGS; Iliya V. Stepanov, PhD, ScD \\ Voronezh State Medical University named after N.N. Burdenko \\ Voronezh, the Russian Federation
}

\begin{abstract}
This article presents clinical cases of successful endodontic treatment of the main types of combined apical and marginal pathology. Based on the results obtained, the optimal method that ensures a successful treatment result for this pathology in one visit, often without subsequent surgical manipulations, is an adequate endodontic mechanical intervention with subsequent obturation of the root canals with a bioceramic sealer, which is confirmed by the literature data. (International Journal of Biomedicine. 2021;11(2):184-187.)
\end{abstract}

Key Words: cone beam computed tomography $\bullet$ root canal $\bullet$ endodontic treatment

For citation: Shumilovich, BR, Podoprigora AV, Sushchenko AV, Rostovtsev VV, Bishtova IS, Stepanov IV. Endodontic Treatment of Periapical Lesions Using Bioceramic Sealers. International Journal of Biomedicine. 2021;11(2):184-187. doi:10.21103/ Article11(2)_CR3

\section{Abbreviations}

CBCT, cone beam computed tomography; MTA, mineral trioxide aggregate; MB2, the second canal of mesiobuccal roots

\section{Introduction}

A number of researchers have noted that today in more than $5.7 \%$ of the teeth in patients over $40-45$ years old with apical periodontal lesions, a comprehensive examination also diagnoses lesions of the marginal periodontium. ${ }^{(1-5)}$ The success of endodontic treatment of such teeth using traditionally used polymer sealers is extremely low. ${ }^{(2,6,7)}$ However, according to the available data, the use of bioceramic sealers that have recently appeared on the market significantly increases the favorable prognosis of endodontic intervention. ${ }^{(6)}$

*Corresponding author: Prof. Anna V. Podoprigora, PhD, ScD. Department of Maxillofacial Surgery, Voronezh State Medical University named after N.N. Burdenko. Voronezh, Russia. E-mail: ovses@yandex.ru
Bioceramics inherently are the most biocompatible material and consist of aluminum oxide, zirconium dioxide, bioactive glass, glass ceramics, composite components and coatings, hydroxyapatite and resorbable calcium phosphates. In dentistry, bioceramics based on calcium phosphates are used to restore bone defects, and are based on calcium silicates and bioaggregates (mineral trioxide aggregate, MTA) to ensure the process of apexification and filling of endodontic perforations. ${ }^{(9-11)}$

Combined apical-marginal lesions develop when the pre-cement is damaged under the influence of bacterial flora from the periodontal sulcus and root canal. ${ }^{(12-14)}$ According to data from studies, the mixed apical-periodontal microflora were found in $21.1 \%$ of all patients with this pathology..$^{(10,13)}$ As a rule, there are three types of periodontal lesions: sinusitis (in cases of the upper jaw), lesions in the furcation of the roots, and lesions of the marginal periodontium. ${ }^{(6)}$ 
In addition, the literature indicates a low predictability of the outcome of the treatment of such lesions. ${ }^{(8,11)}$ Classic treatment protocols involve a surgical impact on the content of the bone defect and the removal of granulation tissue with its subsequent restoration and plastics of the soft tissues of the gums. ${ }^{(15,16)}$ This article presents two clinical cases of endodontic treatment of combined apical-marginal periodontal lesions of the molar.

\section{Case Presentation 1}

A 49-year-old man presented to the dental clinic for tooth 3.7. In his medical history, somatic diseases were absent. The patient indicated previous endodontic treatment was carried out 6-7 years ago. Percussion of the tooth was painful, thermotest showed a lack of sensitivity, the tooth was filled with composite material, the depth of the periodontal sulcus was within normal limits $(<3 \mathrm{~mm})$, except for the distal surface of the tooth. In this area, a periodontal pocket with a depth of $7 \mathrm{~mm}$ was determined.

When analyzing the diagnostic $\mathrm{CBCT}$ scan, a large area of lightening with indistinct contours was visualized, partially passing over to the vestibular and lingual surfaces of the distal root 3.7 (Figure 1, a-c). The root canals were obturated along the entire length; after creating a direct endodontic access, a paste containing eugenol was found in them (Figure 1, d). After discussing all possible options with the patient, we decided on endodontic intervention for the 3.7 tooth as the first stage of a comprehensive treatment plan, followed by periodontal treatment and orthopedic correction of the occlusion state.

Treatment was performed under magnification using an operating microscope. After local anesthesia, the tooth was isolated and an endodontic access cavity was formed. Next, root canals were disinfected using ProTaper Universal Manual S1 and S2 and abundant irrigation with a 3\% sodium hypochlorite solution. Instrumental processing was carried out according to the "long" protocol HyFlex EDM (Coltene, Switzerland) with intermediate active irrigation with a $5 \%$ sodium hypochlorite solution with an endoactivator and EDTA 17\% solution (Figure 1, e).

Protocol (step by step):

- MB - HyFlex 20/05 EDM, 25/ EDM, 30/04 CM, 40/04 EDM, 50/03 EDM. MAF - 50/03;

- ML - HyFlex 20/05 EDM, 25/ EDM, 30/04 CM, 40/04 EDM, 50/03 EDM. MAF - 50/03;

- D - HyFlex 20/05 EDM, 25/ EDM, 40/04 EDM, 50/03 EDM, 60/02 EDM, H-file 70/02. MAF - 70/02.

Root canal obturation was performed with the GuttaFlow bioseal system (Coltene, Roeko, Germany) containing bioglass, according to the one-pin technique (Figure 1, f). The endodontic access cavity was hermetically closed with a composite. Postoperative CBCT showed complete hermetic root canal filling (Figure 1, g-h). On CBCT after 6 months, the regenerative processes in the bone tissue were clearly defined (Figure 1, i). Subsequent X-ray control was performed 12 months after treatment (Figure 1, j): Bone tissue was almost completely restored, the periodontal pocket was not probed, and there were no complaints.

\section{Case Presentation 2}

A 30-year-old woman presented to the dental clinic with a referral from an otolaryngologist. In his medical history, somatic diseases were absent. The causal tooth was 1.6. Local examination: the previous endodontic therapy, the tooth was asymptomatic. CBCT visualized a lightening area with indistinct contours in the periapical region of all roots (Figure 2, a-b). The root canals were obturated along their entire length. When analyzing the diagnostic CBCT, the first cause of the complication was the presence of MB2 (Figure 2, c).

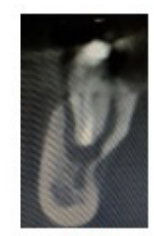

$1, \mathrm{a}$

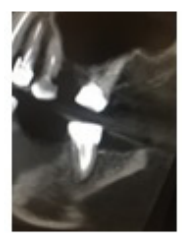

$1, \mathrm{~g}$

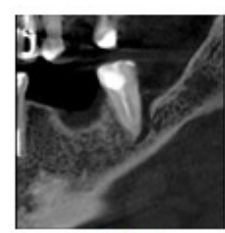

$1, \mathrm{~b}$

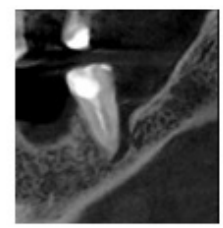

$1, \mathrm{~h}$

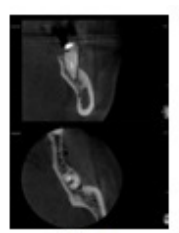

$1, \mathrm{c}$

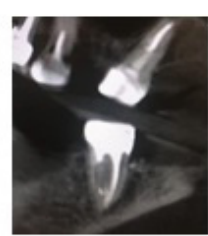

$1, \mathrm{i}$

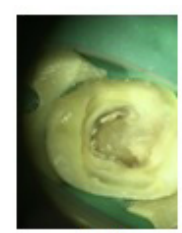

$1, d$

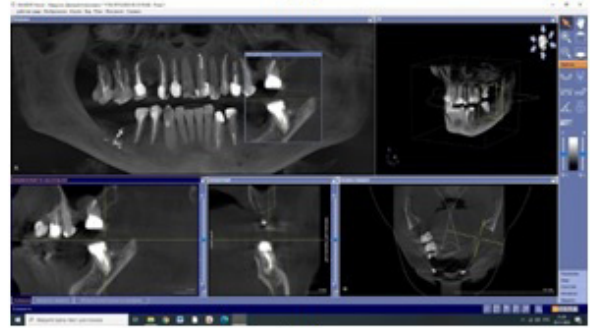

$1, \mathrm{j}$
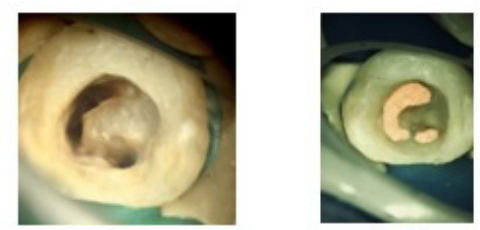

$1, \mathrm{f}$

Fig. 1. a-c) Fragment of CBCT: Initial clinical status (06/26/2019) (tooth 3.7) - the resorption lacuna is visualized as a lightening area with indistinct contours on the distal side of the distal root; d) View of the access cavity, remnants of obturation material; e) View of the cavity after mechanical treatment of root canals; f) View of the cavity after obturation of root canal treatment with GuttaFlow bioseal; $g$-h) Postoperative CBCT demonstrating complete hermetic root canal filling; i) Fragment of CBCT - 6 months after treatment (01/28/2020). j) Fragment of CBCT - 12 months after treatment (06/23/2020). 


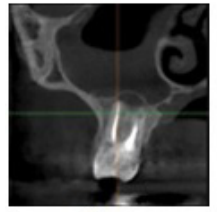

2. a

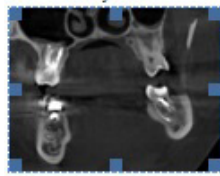

$2, \mathrm{~g}$

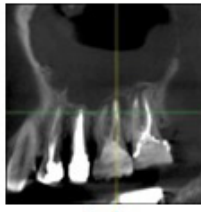

$2, \mathrm{~b}$

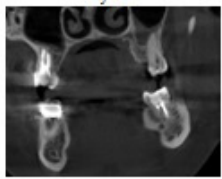

$2, \mathrm{~h}$

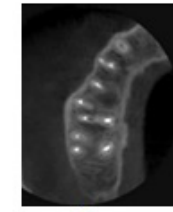

2. c

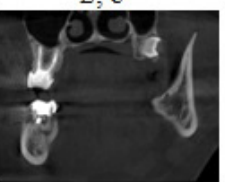

$2, \mathrm{i}$

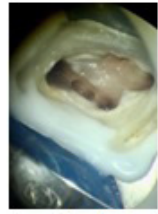

$2, \mathrm{~d}$

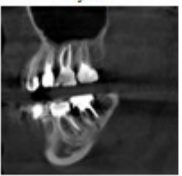

$2, \mathrm{j}$

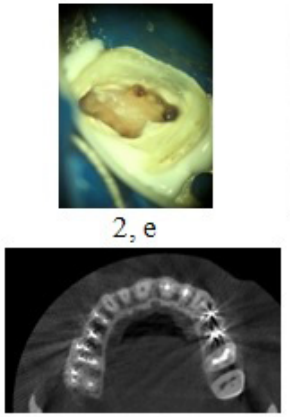

$2, \mathrm{k}$
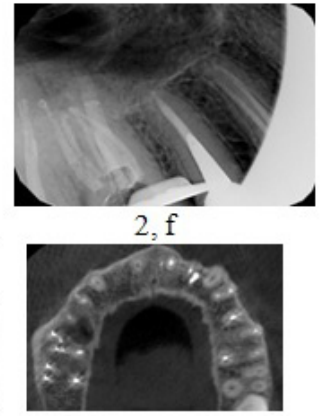

2,1

Fig. 2. a) CBCT (coronal section). The first upper molar on the right (1.6), a "bone" defect and changes in the maxillary sinus are visualized; b) CBCT (lateral section). The first upper molar on the right (1.6), a "bone" defect and changes in the maxillary sinus are visualized; c) CBCT (axial section). The first upper molar on the right (1.6), a "bone" defect is visualized; d-e) Tooth appearance after mechanical treatment of root canals; f) Aiming X-ray of root canal obturation control; g-i) Control CBCT: 11 months after treatment (coronary section); j) Control CBCT: 11 months after treatment (lateral section); $k$-l) Control CBCT: 11 months after treatment (axial section).

However, the radiological picture in the region of the buccal distal canal, where the presence of an additional canal was also suggested, alerted us that there might be further problems..

After discussion with the patient, the decision was made in favor of endodontic treatment. Treatment was performed under magnification using an operating microscope. After local anesthesia, the tooth was isolated and an endodontic access cavity was formed. Then the root canals were disinfected with the Remover 30/07 instrument (MicroMega, Coltene, Switzerland). The clinical picture exceeded all expectations and caused us to make serious adjustments to the existing information about the anatomy of the root canals. A total of 7 root canals were identified (Figure 2, d-e). Then the channels were processed according to the following protocol:

- MB1 - HyFlex EDM 20/05, EDM 25/ , CM 30/04, EDM 40/04. MAF - 40/04;

- MB2 - HyFlex EDM Glidepath 10/05, EDM 15/03, EDM 20/05, EDM 25/ , CM 30/04. MAF - 30/04;

- MB3 - HyFlex EDM Glidepath 10/05, EDM 15/03, EDM 20/05, EDM 25/ . MAF - 25/ ;

- DB1 - HyFlex EDM 20/05, EDM 25/ , CM 30/04, EDM 40/04, EDM 50/03. MAF - 50/03;

- DB2 - HyFlex EDM Glidepath 10/05, EDM 15/03, EDM 20/05, EDM 25/ , CM 30/04, EDM 40/04. MAF 40/04;

- DB3 - HyFlex EDM Glidepath 10/05, EDM 15/03, EDM 20/05, EDM 25/ , CM 30/04. MAF - 30/04;

- Palatinal - HyFlex EDM 20/05, EDM 25/ , EDM 40/04, EDM 50/03, EDM 60/02, H-file 80/02. MAF - $\geq 80 / 02$.

Buccal obturation was performed with GuttaFlow bioseal (Coltene, Roeko, Germany) (Figure 2, f). In the palatal canal, due to significant resorption of the apical part, the apical third of the canal was filled with BioDentin (Dentsply, Switzerland), then GuttaFlow bioseal. On the CBCT fragments after 11 months (Figure 2, g-1), the restoration of bone tissue in the periapical region was verified by the normalization of the X-ray picture in the area of the maxillary sinus, which was confirmed by the otolaryngologist.

\section{Discussion}

This article presents clinical cases of successful endodontic treatment of the main types of combined apical and marginal pathology.

The properties of bioglass were described for the first time in the works of L. Hench. ${ }^{(17,18)}$ Preservation of the original anatomy of the root canal during its preparation plays an extremely important role. ${ }^{(6,19)}$ In all demonstrated clinical cases, new HyFlex EDM files (Coltene, Switzerland) were used for instrumentation. The choice of files was dictated by the basic requirements for a rotary instrument in the treatment of such pathologies:

- A minimum lateral load (i.e. high centering properties and inability to cause the transport of the root canal) so with repeated endodontic intervention, the walls of the root canal are already weakened and there is a danger of "tape" perforations.

- An availability of "large" sizes (> 40 ISO) for effective necrotomy.

HyFlex EDM files are fully compliant with these requirements. In addition, they have a controlled shapememory effect, and at the same time are characterized by a unique combination of the highest flexibility and resistance to breakage. ${ }^{(20)}$ HyFlex EDM NiTi files are manufactured using the EDM method. The working part of the file is processed by the thermal action of pulsed electrical discharges, excited between the electrode-tool and the workpiece, due to which the entire surface of the EDM file is working, and not just the edges as in tools made by the classical milling method. ${ }^{(21)}$ Also, HyFlex EDM files have a changed phase composition and can be deformed due to the reorientation of the formed martensite, which increases their resistance to torsion loads in comparison with other files, including those made using Wire technology. ${ }^{(22)}$

Also, in such clinical situations, it is necessary to emphasize the importance of $\mathrm{CBCT}$, which provides comprehensive information on the location of the resorption defect in relation to periodontal tissues, tooth tissues and a number of anatomical formations (maxillary sinus, mandibular 
canal, etc.). ${ }^{(23,24)}$ It should be noted that, as with any device emitting ionizing radiation, the use of CBCT is justified only if the benefits significantly outweigh the risks. In order to avoid mistakes in diagnosis and harm to the patient, it is important to consider the ALARA principle (As Low As Reasonably Achievable). In accordance with the ALARA concept, the diagnostic effect should certainly be higher than the risk of radiation-induced diseases. ${ }^{(25)}$

Several studies have also suggested the use of calcium hydroxide paste to treat the comorbidity described above over several visits. ${ }^{(2)}$ However, when choosing such a treatment option, care should be taken when introducing material into the area of the defect due to possible mechanical and chemical irritation of the periodontal tissues, which worsens the prognosis of treatment. ${ }^{(8)}$ Thus, an adequate endodontic mechanical intervention followed by root canal obturation with a bioceramic sealer is the optimal method that ensures a successful treatment of combined apical and marginal lesions of the periodontium in one visit, often without subsequent surgical procedures. ${ }^{(6,8,11)}$

\section{Competing Interests}

The authors declare that they have no competing interests.

\section{References}

1. Barros J, Silva MG, Rôças IN, Gonçalves LS, Alves FF, Lopes MA, Pina-Vaz I, Siqueira JF Jr. Antibiofilm effects of endodontic sealers containing quaternary ammonium polyethylenimine nanoparticles. J Endod. 2014 Aug;40(8):1167-71. doi: 10.1016/j.joen.2013.12.021.

2. Tsesis I, Nemcovsky CE, Nissan J, Rosen E. EndodonticPeriodontal Lesions. Evidence-Based Multidisciplinary Clinical Management. Springer; 2019.

3. Amine Kh, El Kholti W, Kissa Ja, Periodontal Root Coverage. An Evidence-Based Guide to Prognosis and Treatment. Springer; 2019.

4. Duncan HF, Cooper PR. Clinical Approaches in Endodontic Regeneration. Current and Emerging Therapeutic Perspectives. Springer; 2019.

5. Peters OA. The Guidebook to Molar Endodontics. Springer; 2017.

6. Jain P. Common Complications in Endodontics. Prevention and Management. Springer; 2018.

7. Hosseinpour S, Walsh LJ, Moharamzadeh K. Regenerative Approaches in Dentistry. An Evidence-Based Perspective, Springer; 2021.

8. Shacham M, Levin A, Shemesh A, Lvovsky A, Ben Itzhak J, Solomonov M. Accuracy and stability of electronic apex locator length measurements in root canals with wide apical foramen: an ex vivo study. BDJ Open. 2020 Nov 17;6(1):22. doi: 10.1038/s41405-020-00052-3.

9. Shumilovich BR, Sushchenko AV, Morozov AN, Kharitonov DU. Comparative clinical and laboratory characteristics of the quality of the filling of root canals using three obturation systems. Dent Oral Craniofac Res. 2015;1(5):160-169.

10. Yilmaz HG, Kalender A, Cengiz E. Use of mineral trioxide aggregate in the treatment of invasive cervical resorption: a case report. J Endod. 2010 Jan;36(1):160-3. doi: 10.1016/j. joen.2009.07.002.

11. Tsesis I, Fuss Z. Diagnosis and treatment of accidental root perforations. Endodontic Topics. 2006;13(1):95-107.

12. Zhou X, Li Yu. Atlas of Oral Microbiology: From Healthy Microflora to Disease. Springer; 2020.

13. Fuss Z, Tsesis I, Lin S. Root resorption--diagnosis, classification and treatment choices based on stimulation factors. Dent Traumatol. 2003 Aug;19(4):175-82. doi: 10.1034/j.1600-9657.2003.00192.x.

14. Tronstad L. Root resorption--etiology, terminology and clinical manifestations. Endod Dent Traumatol. 1988 Dec;4(6):241-52. doi: 10.1111/j.1600-9657.1988.tb00642.x. 15. Heithersay GS. Treatment of invasive cervical resorption: an analysis of results using topical application of trichloracetic acid, curettage, and restoration. Quintessence Int. 1999 Feb;30(2):96-110.

16. Asgary S, Nosrat A. Conservative Management of Class 4 Invasive Cervical Root Resorption Using Calcium-enriched Mixture Cement. J Endod. 2016 Aug;42(8):1291-4. doi: 10.1016/j.joen.2016.05.001.

17. HenchL. Bioceramics. JAmerCeram Soc. 1998;81(7):17051728 .

18. Katsamakis S, Slot DE, Van der Sluis LW, Van der Weijden F. Histological responses of the periodontium to MTA: a systematic review. J Clin Periodontol. 2013 Apr;40(4):334-44. doi: $10.1111 /$ jcpe. 12058 .

19. Wigler R, Koren T, Tsesis I. Evaluation of Root Canal Cleaning and Shaping Efficacy of Three Engine-driven Instruments: SafeSider, ProTaper Universal and Lightspeed LSX. J Contemp Dent Pract. 2015 Nov 1;16(11):910-4. doi: 10.5005/jp-journals-10024-1780.

20. Zupanc J, Vahdat-Pajouh N, Schäfer E. New thermomechanically treated NiTi alloys - a review. Int Endod J. 2018 Oct;51(10):1088-1103. doi: 10.1111/iej.12924.

21. Pirani C, Iacono F, Generali L, Sassatelli P, Nucci C, Lusvarghi L, Gandolfi MG, Prati C. HyFlex EDM: superficial features, metallurgical analysis and fatigue resistance of innovative electro discharge machined NiTi rotary instruments. Int Endod J. 2016 May;49(5):483-93. doi: 10.1111/iej.12470. 22. Iacono F, Pirani C, Generali L, Bolelli G, Sassatelli P, Lusvarghi L, Gandolfi MG, Giorgini L, Prati C. Structural analysis of HyFlex EDM instruments. Int Endod J. 2017 Mar;50(3):303-313. doi: 10.1111/iej.12620.

23. European Society of Endodontology position statement: External Cervical Resorption. European Society of Endodontology (ESE) developed by Dummer PMH, Patel S, Lambrechts P, Shemesh H, Mavridou A. Int Endod J. 2018 Dec;51(12):1323-1326. doi: 10.1111/iej.13008.

24. Rosen E, Taschieri S, Del Fabbro M, Beitlitum I, Tsesis I. The Diagnostic Efficacy of Cone-beam Computed Tomography in Endodontics: A Systematic Review and Analysis by a Hierarchical Model of Efficacy. J Endod. 2015 Jul;41(7):1008-14. doi: 10.1016/j.joen.2015.02.021.

25. Rosen E, Tsesis I. [Use of Cone Beam Computed Tomography in endodontics: rational case selection criteria]. Refuat Hapeh Vehashinayim (1993). 2016 Jan;33(1):35-44, 62. [Article in Hebrew).

26. Baratto-Filho F, Limongi O, Araújo Cde J, Neto MD, Maia SM, Santana D. Treatment of invasive cervical resorption with MTA: case report. Aust Endod J. 2005 Aug;31(2):76-80. doi: 10.1111/j.1747-4477.2005.tb00232.x. 